\title{
REDES NEURAIS ARTIFICIAIS RECORRENTES APLICADAS NA CORREÇÃO DE SINAIS DISTORCIDOS PELA SATURAÇÃO DE TRANSFORMADORES DE CORRENTE
}

\author{
E. C. Segatto* \\ segatto@sel.eesc.sc.usp.br
}

\author{
D. V. Coury* \\ couryesel.eesc.sc.usp.br
}

* Depto de Engenharia Elétrica

EESC - Escola de Engenharia de São Carlos

USP - Universidade de São Paulo

Av. Dr. Carlos de Botelho, 1465

São Carlos - SP - Brasil

\section{RESUMO}

Este trabalho apresenta uma técnica alternativa para a correção de ondas distorcidas provenientes da saturação dos transformadores de corrente (TCs) através de ferramentas inteligentes baseadas em Redes Neurais Artificiais (RNAs) recorrentes. Os TCs estão presentes em Sistemas Elétricos de Potência com a finalidade de proteção e medição, sendo altamente susceptíveis à saturação. O programa EMTP-ATP (Electromagnetic Transients Program) foi escolhido como ferramenta computacional para a simulação de um sistema elétrico utilizado na geração de dados de treinamento e testes para as RNAs. Muitas arquiteturas de redes neurais artificiais foram treinadas e testadas. Resultados promissores relativos ao novo método são apresentados.

PALAVRAS-CHAVE: Saturação, Transformadores de Corrente, Redes Neurais Artificiais, Transformadores de Potência.

\footnotetext{
Artigo submetido em 09/03/2004

1a. Revisão em 28/09/2005

2a. Revisão em 16/08/2006

3a. Revisão em 19/10/2006

Aceito sob recomendação do Editor Associado

Prof. Glauco Nery Taranto
}

\section{ABSTRACT}

This paper presents an alternative approach to the correction of distorted waveforms caused by the current transformer (CT) saturation. This method uses the Artificial Neural Networks (ANNs) recurrent algorithms. Current transformers are present in the electric power systems for protection and measurements and they are highly susceptible to the saturation phenomenon. The EMTP-ATP software has been chosen as the computational tool to simulate the electrical system in order to generate data to train and test the ANNs. Many ANN architectures were trained and tested. Encouraging results related to the application of the new method are presented.

KEYWORDS: Saturation, Current Transformers, Artificial Neural Networks, Power Transformers.

\section{INTRODUÇÃO}

Conforme os Sistemas Elétricos de Potência crescem em tamanho e complexidade, apresentando aumento nos níveis das correntes de falta, os relés de proteção e os equipamentos de medição passam a desempenhar um papel cada vez mais importante. Para tal finalidade, eles requerem uma reprodução precisa das correntes do sistema. Assim, os transformadores de corrente (TCs) são empregados na redução das correntes primárias do sistema. 
A função dos transformadores de corrente, também conhecidos como transdutores, é transformar as correntes oriundas do sistema de potência em valores de baixa magnitude e fornecer isolação galvânica entre a rede elétrica e os relés e outros instrumentos conectados ao enrolamento secundário do TC.

Os ajustes deste enrolamento têm sido padronizados, e um certo grau de intercâmbio entre os fabricantes de relés e medidores pode ser obtido. Em muitos países os enrolamentos secundários dos TCs são ajustados para $5 \mathrm{~A}$, enquanto que na Europa um segundo padrão de ajuste de 1 A é também utilizado. Estes são valores nominais e os transdutores devem ser projetados para tolerar valores maiores oriundos de condições anormais de operação do sistema. Assim, os TCs são concebidos para suportar correntes de falta e outros surtos por poucos segundos, que podem atingir valores de até 50 vezes a magnitude da corrente de carga (Horowitz e Phadke, 1995).

Os TCs, que são magneticamente acoplados e com multienrolamentos, podem ser dispositivos isolados ou estar embutidos nas ramificações de alguns equipamentos de potência, como circuito de disjuntores ou transformadores com aterramento. Dispositivos transdutores mais recentes utilizam tecnologias eletrônicas e componentes de fibra ótica, mas sua aplicação prática ainda não está difundida por questões econômicas.

As correntes fornecidas pelo lado secundário dos transdutores devem ser reproduções fiéis das correspondentes correntes do seu lado primário. Embora os dispositivos modernos atendam a essa condição satisfatoriamente bem, na maior parte dos casos, o projeto de proteção necessita levar em consideração o correto ajuste dos transdutores, bem como os erros de transformação eventualmente introduzidos pelos TCs, de forma que o desempenho dos relés de proteção na presença destes erros possa ser avaliado.

O desempenho dos transdutores sob corrente de carga não é tão preocupante quanto a situação em que o relé deve operar. Quando faltas ocorrem, além dos valores de corrente atingirem níveis elevados, podem conter substanciais parcelas do componente $\mathrm{CC}$ e, ainda, pode existir fluxo remanescente no núcleo do TC. Todos esses fatores podem levar à saturação do núcleo do transformador de corrente e produzir significante distorção na forma de onda secundária de corrente do dispositivo (Horowitz e Phadke, 1995). Um dos grandes problemas enfrentados pelos projetistas de Sistemas Elétricos de Potência reside na existência de situações nas quais os equipamentos elétricos ficam sujeitos à saturação de alguns de seus componentes. Assim, a corrente secundária de um TC pode não representar precisamente sua corrente primária se o dispositivo saturar. Os relés que dependem desta corrente

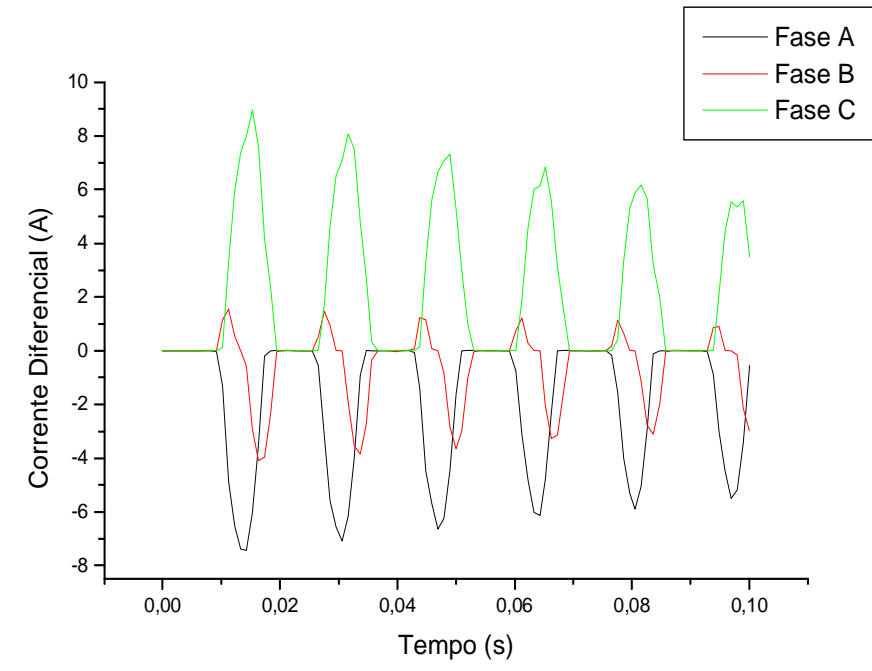

Figura 1: Situação de energização do transformador sem a presença de saturação dos TCs

podem facilmente operar de forma incorreta durante este período, comprometendo a eficiência da proteção do equipamento em questão.

A figura 1 e a figura 2 ilustram a condição de energização de um transformador de potência sem e com a presença de saturação dos TCs respectivamente. Nota-se a diferença no formato de onda do sinal para a mesma situação analisada. Os sinais foram simulados no programa ATP (Alternative Transients Program Rule Book, 1987) e representam as correntes diferenciais em função do tempo, as quais podem ser definidas basicamente como a diferença entre as correntes dos lados primário e secundário do elemento a ser protegido e são fornecidas pelos transformadores de corrente, conectados em ambos os lados do equipamento.

A figura 3 e a figura 4 ilustram uma condição de falta interna ao transformador de potência sem e com a presença de saturação dos TCs respectivamente.

A possibilidade de saturação destes dispositivos deve, portanto, ser altamente considerada no projeto de um sistema de proteção.

Embora muitos trabalhos abordem o problema da saturação dos transformadores de corrente, (Kang et al., 1996; Kang et al., 1997; Jamali and Radji, 1997; Yu et al., 1999; Sidhu et al., 1999; Cummins et al., 2000; Yu et al., 2001), os métodos disponíveis até agora apresentam certas limitações. Engenheiros de proteção têm, portanto, que considerar essa inconveniência quando estabelecerem as especificações em termos do desempenho dos relés.

Este trabalho propõe uma técnica alternativa para a correção 


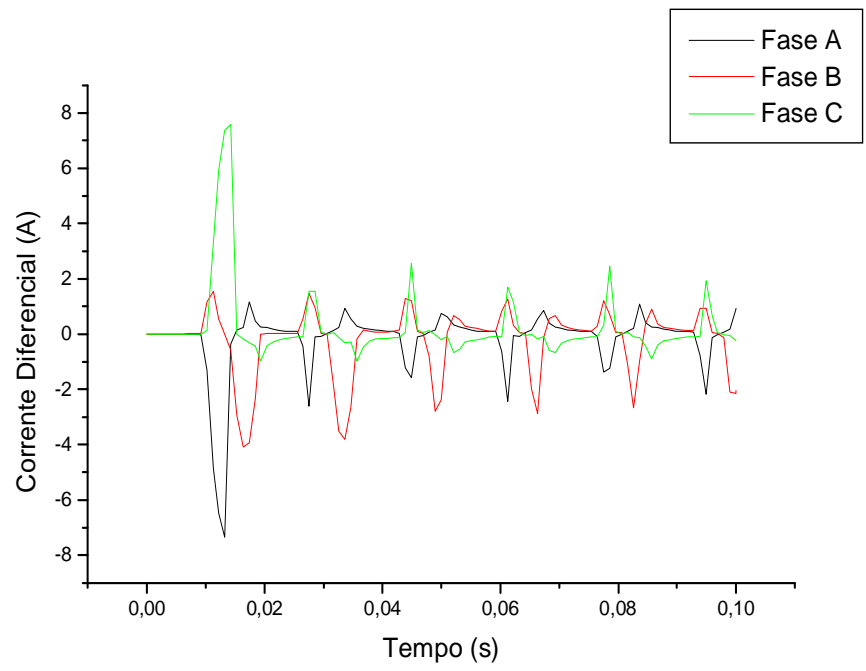

Figura 2: Situação de energização do transformador com a presença de saturação dos TCs

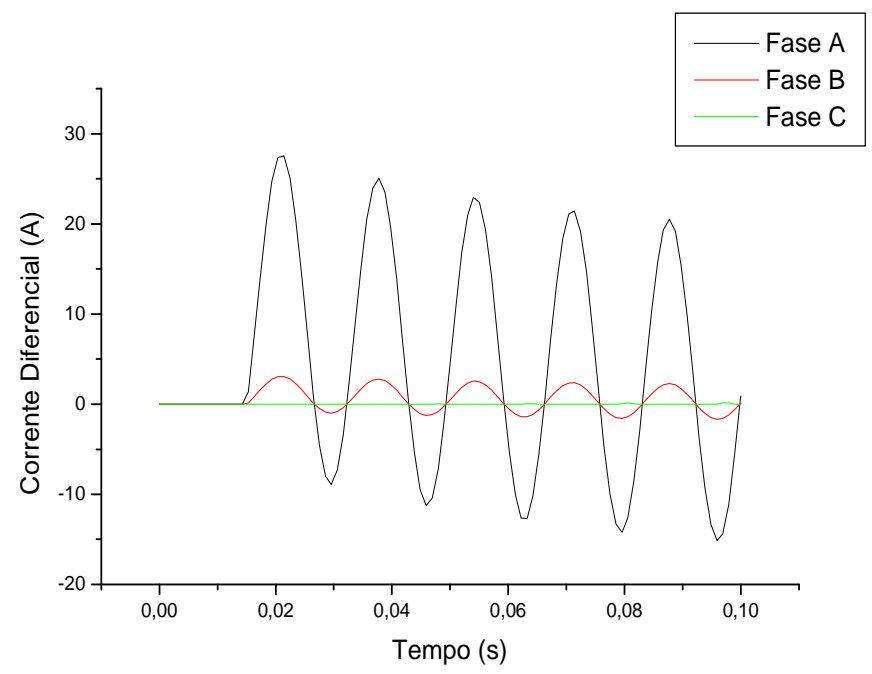

Figura 3: Situação de falta interna ao transformador sem a presença de saturação dos TCs

de formas de ondas distorcidas pela saturação dos TCs baseada no uso de Redes Neurais Artificiais recorrentes. Resultados bastante promissores são alcançados com a utilização desta metodologia e são apresentados a seguir.

\section{A SATURAÇÃO DOS TRANSFORMA- DORES DE CORRENTE}

É bem sabido que, acima do "ponto de joelho" da curva característica de magnetização, a corrente de excitação que flui pelo núcleo aumenta mais rapidamente que sob o "ponto de joelho". Assim, o erro da relação de transformação dos TCs

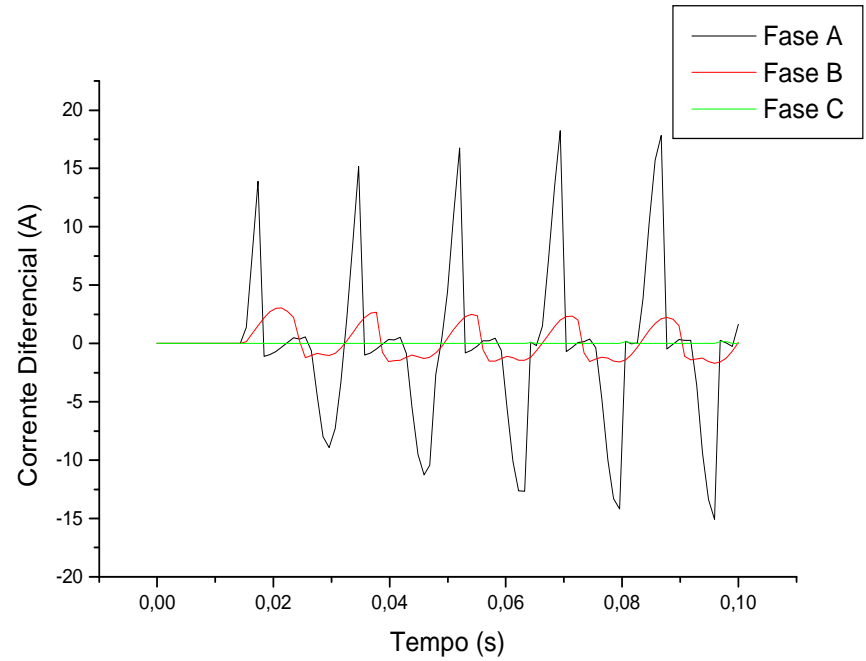

Figura 4: Situação de falta interna ao transformador com a presença de saturação dos TCs

rapidamente se torna mais severo. Isto é chamado de saturação do TC (Powell, 1979), a qual pode causar mal funcionamento dos relés de proteção e equipamentos de medição, conforme explicitado anteriormente.

Na referência (IEEE Power System Relaying Committee, 1997), o tempo de saturação do instante de ocorrência da falta foi padronizado, fornecendo assim os requisitos dos TCs. Baseado nesse critério, a norma ANSI C57.13 (Requirements for Instrument Transformers, 1993) descreve a precisão em termos da carga máxima padrão que pode transportar até 20 vezes a corrente nominal sem exceder $10 \%$ da relação de correção (IEEE, 1991). Isto implica que um aumento na seção transversal do núcleo dos TCs é necessário para permitir uma maior acomodação de fluxo, mas com diminuição na sua sensibilidade.

A presença de fluxo remanescente reduziria o balanço de fluxo disponível em uma direção, tornando a saturação mais provável em condições de falta.

Tem havido muitas propostas para a redução da seção transversal requerida para o TC (Bozoki e Calhoun, 1990), (Masson, 1979) sem afetar a habilidade do dispositivo em lidar com grandes trocas de fluxo. Em (Bozoki e Calhoun, 1990), por exemplo, TCs de núcleo com gap têm sido usados para reduzir o fluxo remanescente e permitir a utilização de seções transversais menores para os núcleos.

Como já citado, uma das principais causas de saturação dos transformadores de corrente é a presença do componente CC na corrente primária. Alguns trabalhos têm reportado a compensação deste componente. Em (Bradley et al., 1978), é proposto um outro circuito capaz de gerar um componente 
CC igual e oposto àquele presente no circuito primário, adicionado ao secundário.

\subsection{Transformadores de Corrente de Nú- cleo de Ferro}

Este tipo de TC é largamente usado para medição de correntes de linha para proteção. Suas vantagens incluem baixo custo, isolação galvânica, confiabilidade e facilidade de implementação. As desvantagens são saturação e fluxo remanescente.

O comportamento do TC sob regime permanente e condições de faltas simétricas é ditado também pela norma ANSI C57.13 (Requirements for Instrument Transformers, 1993). Esse padrão especifica condições para o projeto do TC, as quais incluem: "A tensão terminal secundária do TC é a tensão que o TC fornecerá à uma carga padrão em 20 vezes a corrente secundária nominal sem exceder $10 \%$ da relação de correção. Ainda, a correção da relação deverá ser limitada a $10 \%$ em qualquer corrente de 1 a 20 vezes a corrente secundária nominal na carga padrão ou qualquer carga padrão menor usada para tensões terminais secundárias."

Entretanto, faltas em sistemas de potência envolvendo componentes $\mathrm{CC}$ significantes e superpostos às correntes simétricas (correntes de faltas assimétricas) podem saturar os TCs em correntes muito menores do que correntes simétricas sem componente CC.

Um método de se evitar a saturação do TC é aumentar o tamanho do seu núcleo. Um outro método é usar um material na construção do núcleo que suporte grandes densidades de fluxo. Ambas as opções podem afetar o custo e as facilidades de aplicação do transformador de corrente. Em muitos projetos é desejável incorporar TCs como parte integral de religadores e disjuntores. Aumentar a área do núcleo significa aumentar o diâmetro externo, o qual apresenta dificuldades mecânicas, ou diminuir o diâmetro interno, o que pode afetar os níveis de isolação.

Em muitos casos a correção da saturação do TC usando Redes Neurais Artificiais pode ser implementada nos processadores digitais de sinais existentes e não acrescenta nenhum custo ao controle ou ao relé.

\subsection{Efeitos da Saturação do TC na Opera- ção do Relé}

Quando TCs com núcleo de ferro são usados em sistemas de proteção, o problema de saturação pode se tornar crítico. A maioria dos relés de proteção (eletromecânicos e eletrônicos) tomam as decisões de operação baseados no valor RMS da corrente de falta. Se o sinal fornecido pelo TC é distorcido por saturação, o valor RMS detectado será muito menor que o valor real de corrente falta, podendo atrasar a operação do relé. Quando os TCs não saturam, as correntes secundárias são uma réplica precisa das correntes primárias. Quando a saturação é elevada, sua presença pode até mesmo evitar que o relé opere. Este tipo de problema tem sido amplamente discutido na literatura relativos a vários tipos de proteção (Wright, 1968; Bozoki e Calhoun, 1990; Chen e Glad, 1991; Kezunovic et al., 1993; Barnett et al., 1993; Glinkowski e Esztergalyos, 1996; Kang et al., 1997).

\subsection{Estimadores Existentes para a Corre- ção da Saturação dos TCs}

Em (Conrad et al., 1984), um método para correção das correntes secundárias distorcidas dos TCs foi apresentado assumindo-se que a corrente de magnetização é fornecida explicitamente no cálculo. Porém, como essa corrente não pode normalmente ser fornecida para todos os casos, dificuldades práticas podem surgir.

O trabalho de (Kang et al., 1997) apresenta uma técnica de compensação que estima a corrente secundária correspondente à relação do TC, em particular quando o TC está saturado com ou sem a presença de fluxo remanescente. Os resultados demonstram a habilidade da técnica desenvolvida em fornecer boa precisão na estimativa da corrente secundária ( $<1,5 \%$ de erro ) na presença de saturação do TC. Porém, a estimativa das correntes secundárias é feita através de análise matemática.

Se a característica da saturação do TC é conhecida, uma estimativa da corrente de entrada na situação onde o TC satura pode ser encontrada, assumindo-se carga resistiva (Barnett et al., 1993). A maioria das correntes de falta não terá a característica simétrica necessária para essa estimativa simplificada. Dependendo da impedância da linha e do ângulo de falta, a corrente de falta terá um decaimento exponencial.

Outras abordagens presentes na literatura têm usado filtros de Kalman e processos interativos para a correção da saturação (Chen e Glad, 1991; Kang et al., 1997). Estas abordagens requerem que uma função seja desenvolvida para aproximar características não-lineares do núcleo. Uma estimativa é feita para o valor do fluxo do núcleo baseada na corrente secundária medida. Usando a estimativa do fluxo, é feita uma estimativa da corrente de magnetização. Esta corrente é a diferença entre a corrente secundária medida e a corrente real. Estes métodos não são convenientes para aplicações em tempo real.

Os esquemas de (Mikrut et al., 1989; Hosemann e Steigerwald, 1993) que tratam do fenômeno da saturação dos TCs foram reportados no passado e requerem critérios es- 
peciais para sua correta implementação.

Alguns trabalhos têm sido realizados na tentativa de reconstrução dos sinais distorcidos pelos TCs utilizando Redes Neurais Artificiais. Estas publicações envolvem apenas a estimação das correntes primárias dos TCs, como as técnicas propostas em (Pihler et al., 1997; Yu et al., 1999; Cummins et al., 2000).

Em (Pihler et al., 1997), a rede neural foi aplicada para detecção de correntes de inrush, como também na reconstrução das correntes secundárias do TC distorcidas pela saturação do mesmo, melhorando a confiabilidade da operação de proteção. Os resultados confirmaram reconhecimento rápido e seguro das correntes de magnetização, assim como a reconstrução satisfatória das correntes do TC.

A técnica proposta em (Yu et al., 2001) permanece estável durante as condições de saturação e de erro nas relações dos TCs, porém a técnica descrita utiliza somente dados saturados de falta na etapa de treinamento das RNAs. As demais situações devem ser interpretadas pelo algoritmo sem a presença da rede neural.

Assim como em (Yu et al., 2001), o trabalho apresentado por (Yu et al., 1999) emprega dois conjuntos de coeficientes para as RNAs a fim de lidar com diferentes faixas de correntes de falta.

Em (Cummins et al., 2000), redes neurais artificiais foram treinadas para obter a função de transferência inversa dos TCs toroidais e estimar as correntes secundárias distorcidas pela saturação dos mesmos. A velocidade e a precisão da RNA obtida mostraram-se satisfatórias em aplicações em tempo-real.

A maioria dos algoritmos propostos para este tipo de proteção, no passado, usava critérios especiais para a detecção da saturação e atrasavam a operação dos relés para evitar operações incorretas.

\section{REDES NEURAIS ARTIFICIAIS}

A rede de neurônios artificiais foi inspirada no pouco que o homem já conhece sobre o cérebro humano. O mesmo possui as características de robustez, tolerância à falhas, capacidade de ajuste a novos ambientes pelo treinamento, potencial para processar informações ruidosas, além de alta velocidade de processamento. As transmissões e recepções entre neurônios acontecem por aumento ou diminuição do potencial elétrico na célula receptora. $O$ primeiro modelo artificial de um neurônio biológico foi fruto do trabalho pioneiro de Warren McCulloch e Walter Pitts (McCulloch e Pitts, 1943). Em 1958, Frank Rosenblatt demonstrou seu modelo chamado Perceptron, no qual, se fossem acrescidas de ligações ajustá- veis, as RNAs poderiam ser treinadas para classificar certos tipos de padrões, propondo ainda um algoritmo para treinar a rede e executar determinados tipos de funções (Rosenblatt, 1958). Em 1969, Minsky e Papert chamaram a atenção para algumas tarefas que os atuais modelos não eram capazes de realizar, já que só resolviam problemas linearmente separáveis (Minsky e Papert, 1969). A descrição do algoritmo de treinamento de retropropagação (Rumelhart, Hinton e Williams, 1986), mostrou que as visões anteriores sobre o Perceptron eram bastante pessimistas. As RNAs de múltiplas camadas são, sem dúvida, capazes de resolver problemas "difíceis de aprender". A partir de meados da década de 80 houve nova explosão de interesse pelas redes neurais artificiais na comunidade internacional.

Uma RNA completa é formada por um conjunto de neurônios dispostos em uma ou mais camadas, conectados por ligações de pesos variados. A rede Feedforward tem a propagação do sinal para frente, entre células de camadas adjacentes, até a última camada (saída) (SNNS User Manual, 1995). Na fase de treinamento da rede, há a determinação e a correção dos pesos e dos bias, para que a mesma responda de forma desejada, minimizando o erro de saída para cada vetor de entrada. A estrutura de processamento paralelo de informação permite a inclusão de conhecimento hábil no processo, a detecção e a classificação de sinais. A característica da RNA é, então, considerar o conhecimento adquirido durante o treinamento e responder a novos dados de entrada da maneira mais apropriada, concluindo assim, uma generalização do problema.

\subsection{Redes Neurais Recorrentes}

Muitos algoritmos de treinamento das RNAs não são capazes de implementar mapeamentos dinâmicos, como por exemplo o algoritmo de retropropagação simples, que pode apenas aprender mapeamentos estáticos. Um artifício utilizado para processamento temporal utilizando estas redes envolve o uso de janelas de tempo, onde a entrada da rede utiliza trechos dos dados temporais como se eles formassem um padrão estático.

Entretanto, esta solução não é a mais indicada para o processamento temporal. A principal questão, portanto, é como estender a estrutura das redes MLP - Multi Layer Perceptron - para que assumam um comportamento que varie com o tempo, sendo assim capaz de tratar sinais temporais (Carvalho et al., 1998).

O tempo pode ser representado pelo efeito que acarreta no processamento de sinais, que significa oferecer características dinâmicas ao mapeamento realizado pela rede, tornandoa sensível a sinais que variem com o tempo. Para uma RNA ser considerada dinâmica, é preciso que possua memória (El- 
man, 1990).

Existem basicamente duas maneiras de prover memória a uma RNA. A primeira seria introduzindo atraso no tempo, como as técnicas TDNN - Time Delay Neural Network, e FIR Multilayer Perceptron. A segunda maneira e que foi abordada neste trabalho, seria a utilização de redes neurais recorrentes, tais como Backpropagation Through Time, RealTime Recurrent Learning, Cascate Correlation recorrente, redes de Elman e redes de Jordan (Carvalho et al., 1998).

Pode-se definir redes recorrentes como aquelas que possuem conexões de realimentação que proporcionam comportamento dinâmico. Há dois tipos de redes recorrentes: aquelas em que o padrão de entrada é fixo e a saída caminha, dinamicamente, para um estado estável e aquelas em que ambas, entrada e saída, variam com o tempo, sendo estas últimas mais gerais e utilizadas com maior frequiência.

Há muitas variações de arquiteturas de redes recorrentes, sendo que algumas delas permitem o uso de algoritmos de treinamento mais simples ou adaptados a uma tarefa particular. Duas maneiras que podem ser usadas para treinar uma rede recorrente, e que não envolvem o uso de aproximações na computação dos gradientes são Backpropagation Through Time e redes recorrentes de tempo real.

\subsubsection{Redes de Elman e Jordan}

Elman introduziu memória às RNAs (Elman, 1990). Nas redes de Elman, além das unidades de entrada, intermediárias e de saída, há também unidades de contexto, como nas redes parcialmente recorrentes em geral. As unidades de entrada e saída interagem com o ambiente externo, enquanto as unidades intermediárias e de contexto não o fazem. As unidades de entrada são apenas unidades de armazenamento (buffer) que passam os sinais sem modificá-los. As unidades de saída são unidades lineares que somam os sinais que recebem. As unidades intermediárias podem ter funções de ativação lineares ou não lineares, e as unidades de contexto são usadas apenas para memorizar as ativações anteriores das unidades intermediárias e podem ser consideradas como atraso no tempo em um passo.

As conexões feedforward são modificáveis e as conexões recorrentes são fixas, motivo pelo qual a rede de Elman é apenas parcialmente recorrente.

A Figura 5 ilustra o modo de operação de uma rede de Elman.

Em um intervalo de tempo específico k, as ativações das unidades intermediárias (em k-1) e as entradas correntes (em k) são utilizadas como entradas da rede. Em um primeiro estágio, feedforward, estas entradas são propagadas para frente a fim de produzir as saídas. Posteriormente, a rede é trei-

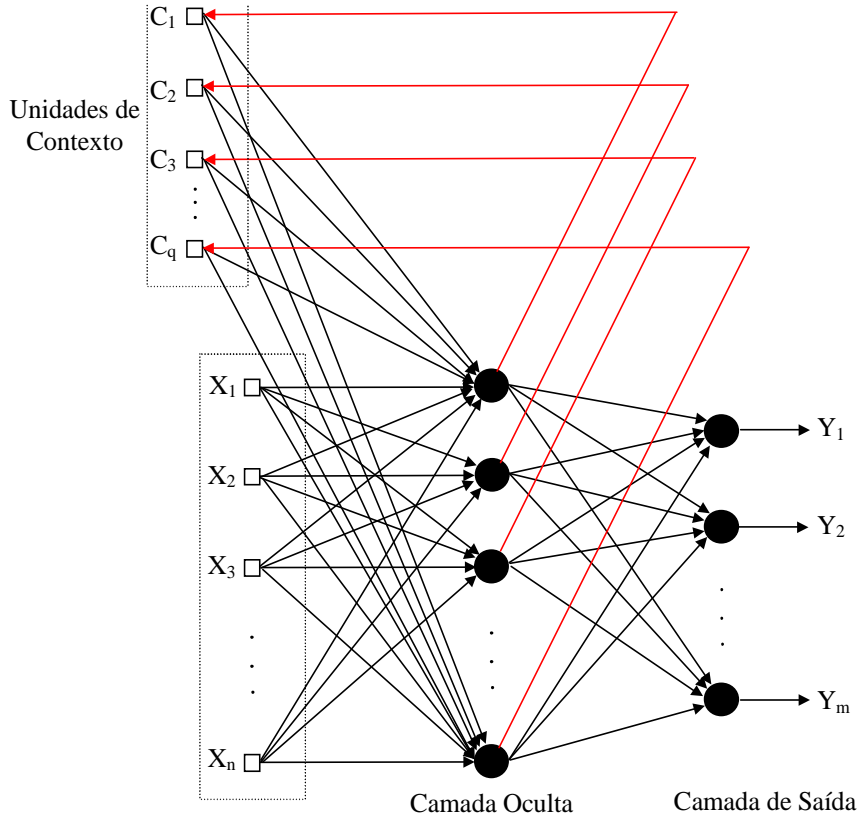

Figura 5: Rede Neural de Elman

nada com o algoritmo de aprendizagem de retropropagação padrão. Após este passo de treinamento, as ativações das unidades intermediárias no tempo k são reintroduzidas através das ligações recorrentes nas unidades de contexto, sendo salvas nestas unidades para o próximo passo do treinamento $(\mathrm{k}+1)$.

No início do treinamento, as ativações das unidades intermediárias são desconhecidas e, geralmente, são inicializadas para a metade do valor máximo que as unidades intermediárias podem ter.

Na rede de Jordan (Jordan, 1986), a saída da rede é copiada para a unidade de contexto. Adicionalmente, as unidades de contexto são localmente recorrentes. A grande diferença em termos de topologia entre as duas redes é que a recorrência na rede de Elman é feita da camada oculta para as entradas, enquanto que na rede de Jordan a recorrência é feita das saídas para as entradas.

As redes neurais recorrentes foram utilizadas em trabalhos que abordam seu emprego no auxílio à tarefa de proteção de sistemas elétricos, como em (Segatto e Coury, 2005; Segatto e Coury, 2006). 


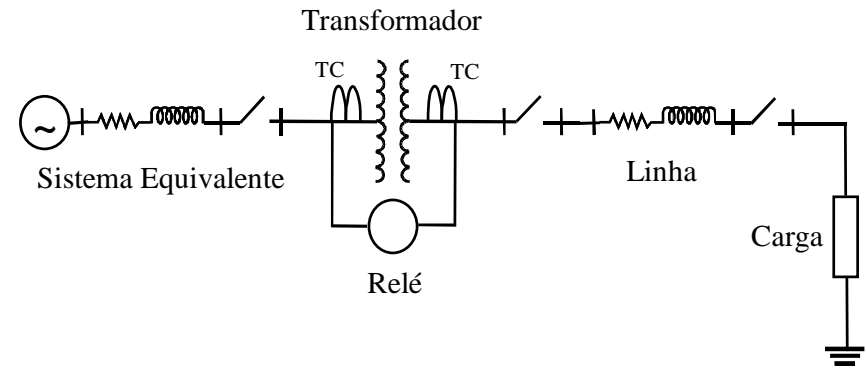

Figura 6: Sistema elétrico simulado no EMTP-ATP

Tabela 1: Valores de fluxo e corrente da curva de saturação do transformador

\begin{tabular}{|c|c|}
\hline Fluxo (V .s) & Corrente (A) \\
\hline 26,8995372 & 1,06055811 \\
29,8883747 & 3,28814356 \\
32,8772122 & 20,8489263 \\
34,3716309 & 129,055289 \\
35,2682821 & 380,764491 \\
\hline
\end{tabular}

\section{A SIMULAÇÃO DO SISTEMA ELÉTRICO ESTUDADO}

\subsection{Aquisição de Dados}

A figura 6 mostra a representação do sistema elétrico simulado pelo programa EMTP-ATP - Electromagnetic Transients Program (Coury et al., 1998; Alternative Transients Program Rule Book, 1987), com o intuito de geração do banco de dados utilizado no treinamento e testes das RNAs.

O sistema é composto de um equivalente de geração de 138 $\mathrm{kV}$ e 30 MVA, um transformador de $138 / 13,8 \mathrm{kV}$ e 25 MVA, uma linha de transmissão de $5 \mathrm{~km}$ seguida de uma carga de 10 MVA e fator de potência 0,92 indutivo.

O transformador trifásico é conectado em configuração delta/estrela e seu modelo no EMTP-ATP foi implementado utilizando-se três transformadores monofásicos.

O modelo utilizado para representar faltas internas é ilustrado na figura 7. As simulações de falta são obtidas dividindo-se os enrolamentos e as impedâncias de acordo com as porções onde as faltas seriam aplicadas.

A tabela 1 apresenta os valores relativos à curva de saturação utilizada na simulação do transformador de potência, fornecida pela CPFL - Companhia Paulista de Força e Luz, correspondente ao transformador utilizado.

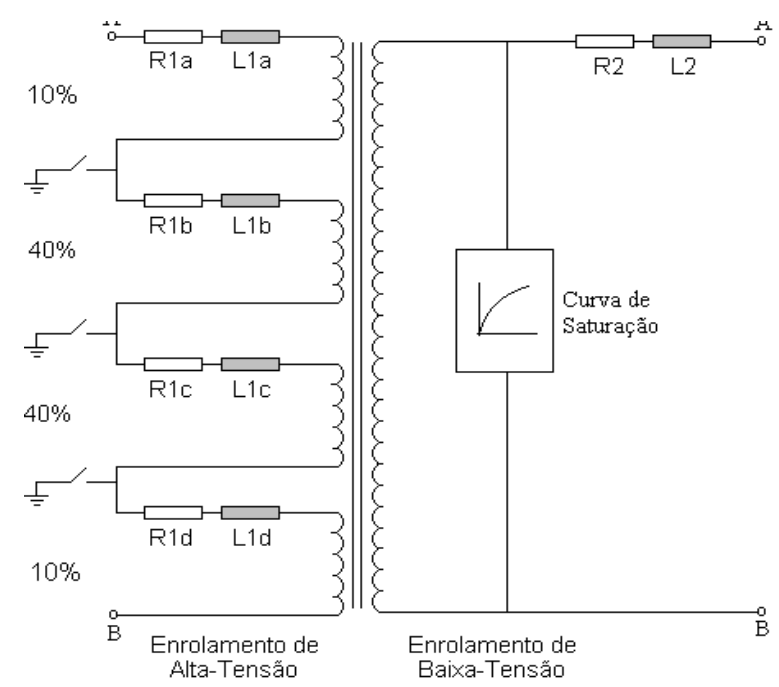

Figura 7: Conexão do enrolamento sob falta

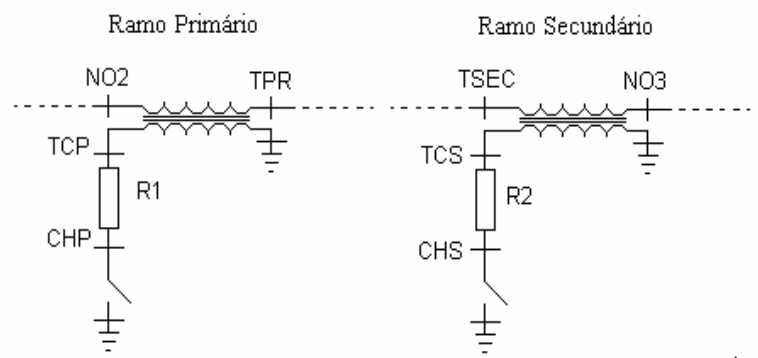

Figura 8: Conexão dos TCs ao sistema elétrico

A figura 8 representa o esquema de conexão dos dispositivos transformadores de corrente ao sistema elétrico através do simulador ATP.

A figura 9 seguinte ilustra a curva de saturação utilizada nas simulações dos transformadores de corrente, fornecida pela CPFL.

A tabela 2 mostra a combinação de valores para os casos de falta interna simulados com o objetivo de treinar a RNA. Estes casos envolveram situações de faltas internas de espiras a terra e também de faltas internas entre espiras. A tabela 3 ilustra esta combinação para os casos de energização. Foram simulados 270 casos de energização, 162 casos de faltas internas de espira para terra $(10,50$ e $90 \%$ do enrolamento envolvido) e 162 casos de faltas internas entre espiras (10, 30 e $70 \%$ do enrolamento envolvido), formando um banco de dados de 1782 vetores, empregados com uma janela de dados móveis de três movimentos.

Após o processo de simulação no programa EMTP-ATP, os dados foram organizados a fim de serem empregados na 


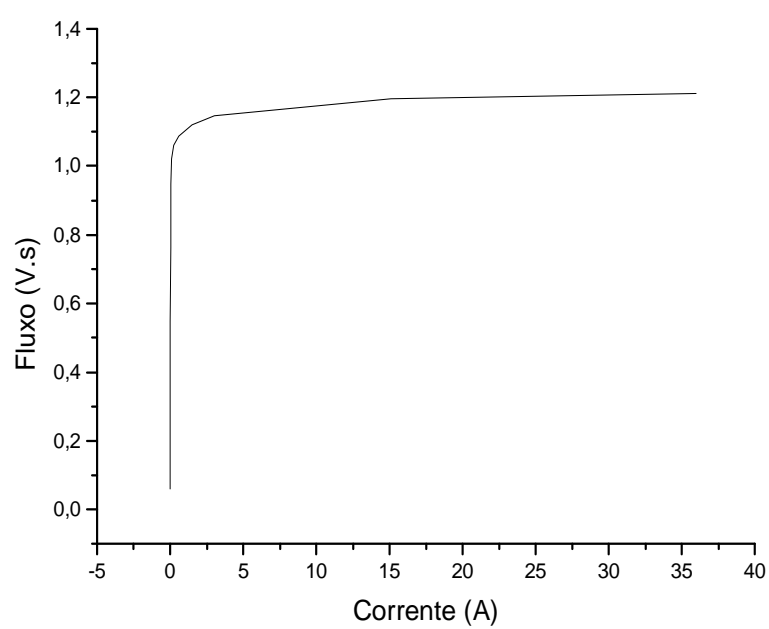

Figura 9: Curva de saturação fornecida para os TCs

Tabela 2: Parâmetros variados nos casos de falta interna simulados

\begin{tabular}{|c|c|c|c|}
\hline Tensão (kV) & $\begin{array}{c}\text { Carga } \\
\text { (MVA) }\end{array}$ & $\begin{array}{c}\% \text { do } \\
\text { enrolamento } \\
\text { envolvido } \\
\text { na aplicação } \\
\text { da falta }\end{array}$ & $\begin{array}{c}\text { Ângulo de } \\
\text { Incepção } \\
\text { (graus) }\end{array}$ \\
\hline 112,7 & 8,0 & 10 & 43,0 \\
115,0 & 10,0 & 30 & 65,0 \\
& 12,0 & 50 & 108,0 \\
& & 70 & 173,0 \\
& & 90 & 216,0 \\
& & & 238,0 \\
& & 260,0 \\
\end{tabular}

etapa de treinamento das RNAs, descrita mais detalhadamente na próxima seção.

\section{REDES NEURAIS NA CORREÇÃO DE ONDAS DISTORCIDAS PROVENIENTES DA SATURAÇÃO DE TCS}

O programa SNNS - Stuttgart Neural Network Simulator (SNNS User Manual, 1995) foi utilizado para treinar e testar as diferentes arquiteturas, a fim de corrigir os sinais distorcidos pela saturação dos TCs. O simulador SNNS foi escolhido devido à sua comprovada eficiência e simplicidade, bem como a amigável interface gráfica.
Tabela 3: Parâmetros variados nos casos de energização simulados

\begin{tabular}{|c|c|c|c|}
\hline \multirow{2}{*}{ Tensão $(\mathrm{kV})$} & \multicolumn{3}{|c|}{ Instante de fechamento das chaves (ms) } \\
\cline { 2 - 4 } & Fase A & Fase B & Fase C \\
\hline 107,0 & 6 & 6 & 6 \\
108,0 & 6 & 6 & 10 \\
109,0 & 6 & 7 & 6 \\
109,5 & 6 & 7 & 10 \\
110,0 & 6 & 9 & 12 \\
111,0 & 6 & 11 & 15 \\
112,7 & 7 & 9 & 11 \\
113,3 & 7 & 11 & 7 \\
114,0 & 7 & 11 & 12 \\
115,0 & 7 & 6 & 9 \\
115,5 & 7 & 7 & 6 \\
116,0 & 7 & 7 & 7 \\
116,5 & 9 & 9 & 13 \\
117,0 & 9 & 10 & 13 \\
118,0 & 9 & 6 & 6 \\
& 9 & 9 & 12 \\
& 9 & 7 & 10 \\
& 9 & 11 & 7 \\
\hline
\end{tabular}

As redes neurais recorrentes foram escolhidas para a tarefa de reconstrução de sinais distorcidos por apresentarem melhor desempenho em relação aos outros tipos de redes neurais estudadas (RBF - Radial Basis Function e MLP).

\subsection{O Processo de Treinamento}

Após a simulação dos dados, estes foram divididos em conjuntos de treinamento, validação e testes. Um total de quatro amostras por fase das correntes diferenciais foram utilizadas no treinamento com freqüência amostral de $1 \mathrm{kHz}$. Ainda, para cada fase, uma janela de dados móveis foi empregada.

As três janelas de dados móveis para a fase A são:

Primeira janela $-\mathrm{i}_{A 1}, \mathrm{i}_{A 2}, \mathrm{i}_{A 3}, \mathrm{i}_{A 4}$

Segunda janela $-\mathrm{i}_{A 2}, \mathrm{i}_{A 3}, \mathrm{i}_{A 4}, \mathrm{i}_{A 5}$

Terceira janela $-\mathrm{i}_{A 3}, \mathrm{i}_{A 4}, \mathrm{i}_{A 5}, \mathrm{i}_{A 6}$

onde $\mathrm{i}_{A 1}, \mathrm{i}_{A 2}, \mathrm{i}_{A 3}, \mathrm{i}_{A 4}, \mathrm{i}_{A 5}$ e $\mathrm{i}_{A 6}$ são os valores discretos de 


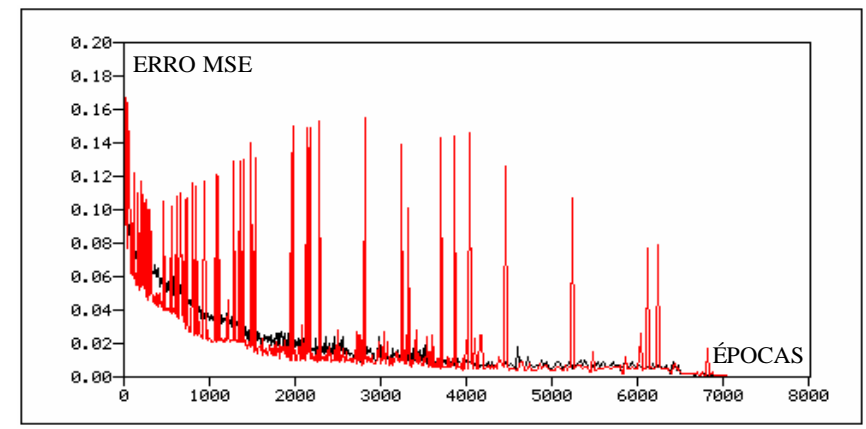

Figura 10: Curva de decaimento dos erros de treinamento e validação da rede 24+20+24 tipo Elman

corrente diferencial da fase A.

Após a adequação dos vetores ao simulador SNNS, executou-se o processo de treinamento da rede neural que conseguiu seu menor erro após 7000 épocas, onde atingiu a média quadrática de erros no valor de 0,0005. Nesta fase, empregaram-se 1600 vetores, onde 800 foram usados no treinamento e 800 na fase de validação.

Destaca-se que, durante esta etapa, foi necessária a realização de uma adaptação na função de transferência dos neurônios. Desta forma, a função Sigmóide Logística presente inicialmente nessas células foi substituída pela função Tangente Hiperbólica a fim de que as redes neurais pudessem realizar cálculos precisos com valores numéricos negativos.

As camadas de entrada e saída das redes neurais contiveram em cada uma 24 dados, pois foram utilizados os 8 primeiros pontos (meio ciclo de dados) de cada uma das 3 fases dos sinais diferenciais.

Para tal resultado, a melhor topologia de rede neural obtida foi a 24+20+24 do tipo Elman, ou seja, 24 neurônios na camada de entrada, 20 neurônios na camada oculta, 24 neurônios na camada recorrente e 24 células na camada de saída, a qual reconstruiu o sinal.

Este procedimento de treinamento é executado apenas uma vez e uma capacidade de generalização da RNA é esperada.

A figura 10 ilustra a curva de decaimento dos erros médios quadráticos (MSE) de treinamento (na cor preta) e validação (na cor vermelha) da rede, após 7000 épocas.

\subsection{Resultados Obtidos}

$\mathrm{Na}$ fase de testes da rede neural melhor classificada na tarefa de reconstrução dos sinais distorcidos, utilizou-se um conjunto de dados com 182 vetores, diferentes daqueles empregados nas fases de treinamento e validação.

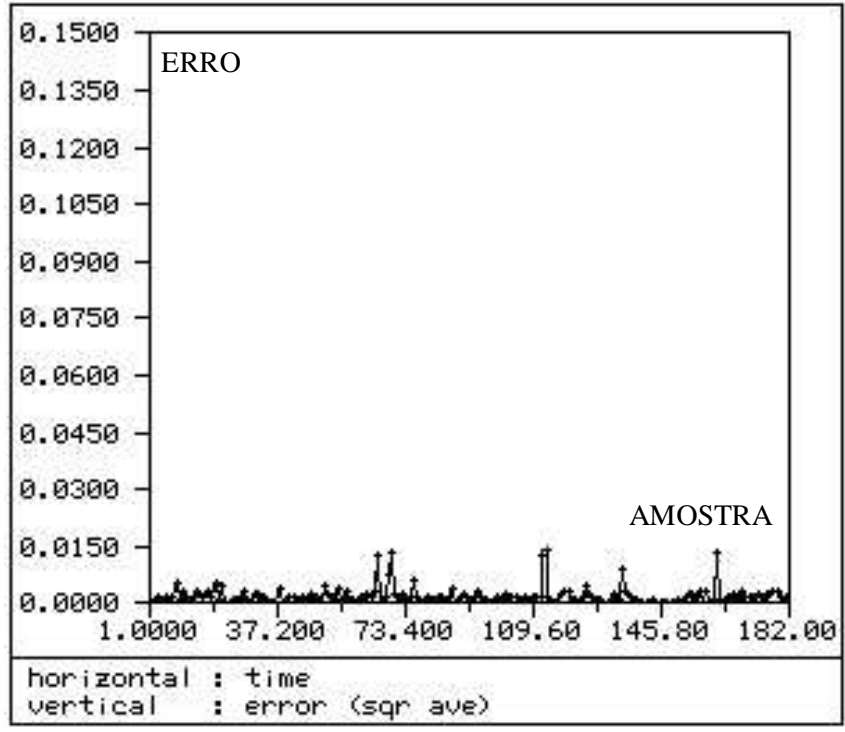

Figura 11: Erros dos testes efetuados na rede $24+20+24$

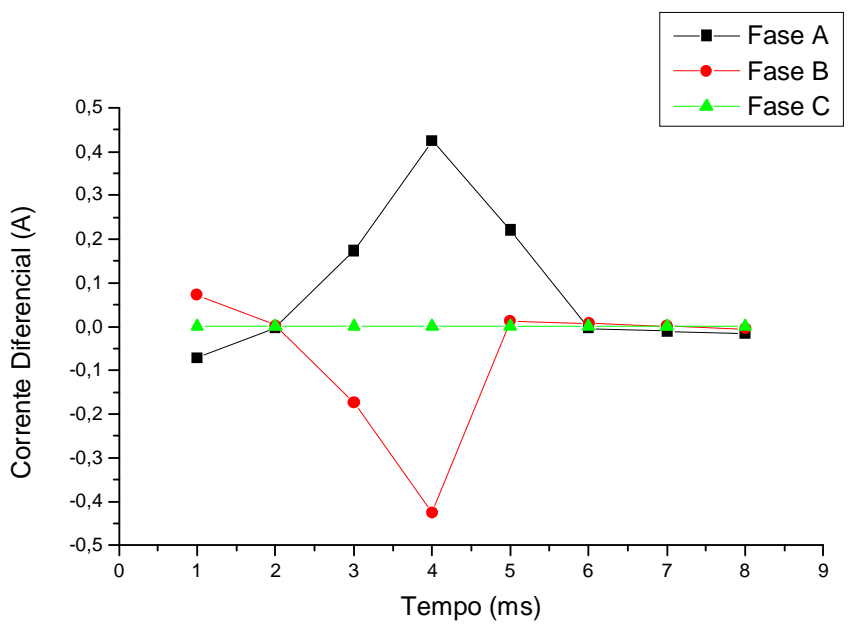

Figura 12: Sinal de energização distorcido por saturação

A figura 11 mostra o gráfico da média dos erros quadráticos de todos os testes efetuados na rede neural de topologia $24+20+24$.

Destaca-se que as redes neurais obtiveram um índice de acerto médio da ordem de $98,5 \%$.

Para a confirmação dos resultados obtidos nos testes da RNAs obtidas, são mostrados a seguir os gráficos dos sinais obtidos através da correção efetuada pela rede neural. A figura 12 mostra o sinal de um caso de energização do transformador contendo distorção causada pela saturação do TC primário.

Na figura 13, é ilustrado o sinal ideal desejado para o mesmo 


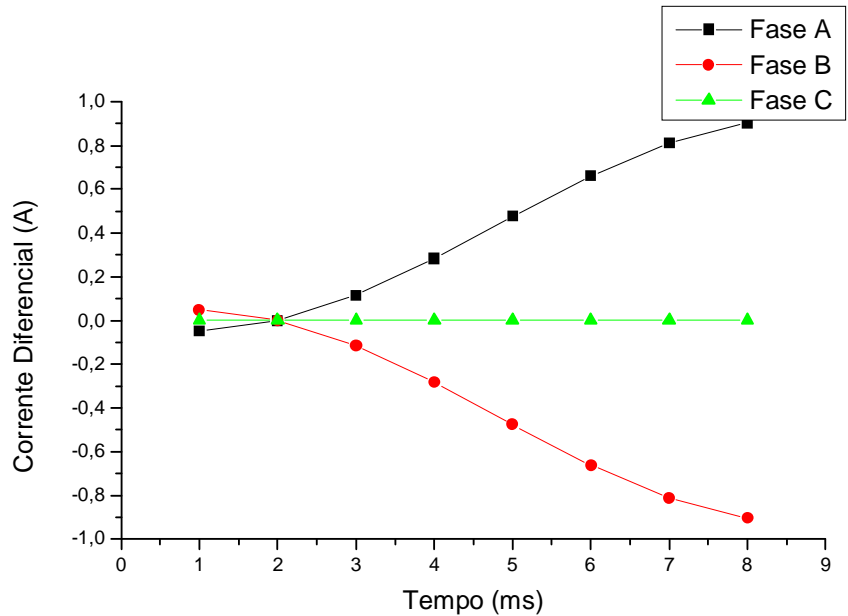

Figura 13: Sinal de energização com TC ideal

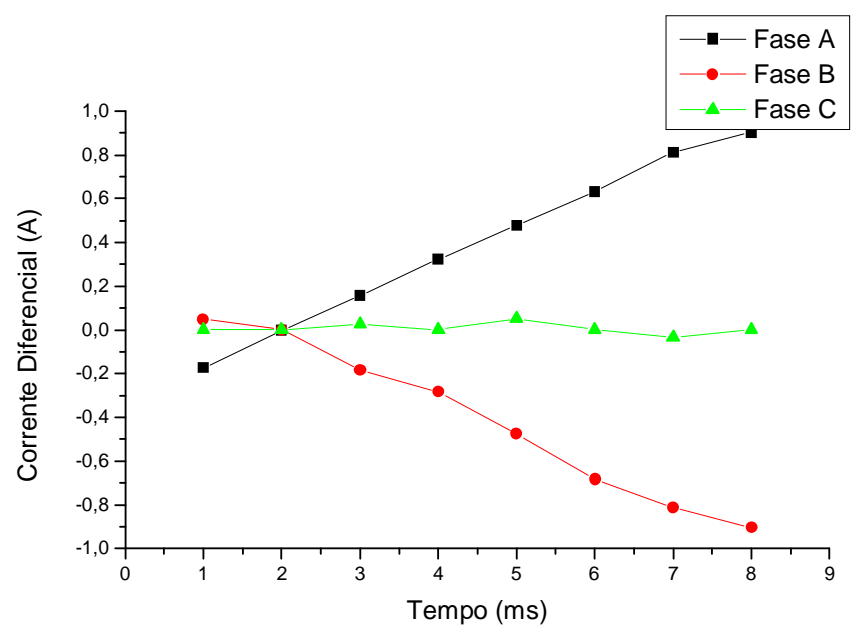

Figura 14: Sinal de energização reconstruído pela RNA

caso, ou seja, sem conter a saturação do TC.

Na figura 14, pode-se observar o caso em questão corrigido pela rede neural recorrente. Salienta-se que os sinais das 3 fases de cada figura foram tomados em sincronismo.

As figuras 15, 16 e 17 mostram situações análogas às anteriores, porém para um caso de falta interna ao transformador.

A Figura 18 mostra o sinal de um outro caso de falta interna ao transformador contendo distorção causada pela saturação do TC primário.

Na Figura 19, é ilustrado o sinal ideal desejado para o mesmo caso, ou seja, sem conter a saturação do transformador de corrente.

Na Figura 20, observa-se o sinal corrigido pela RNA.

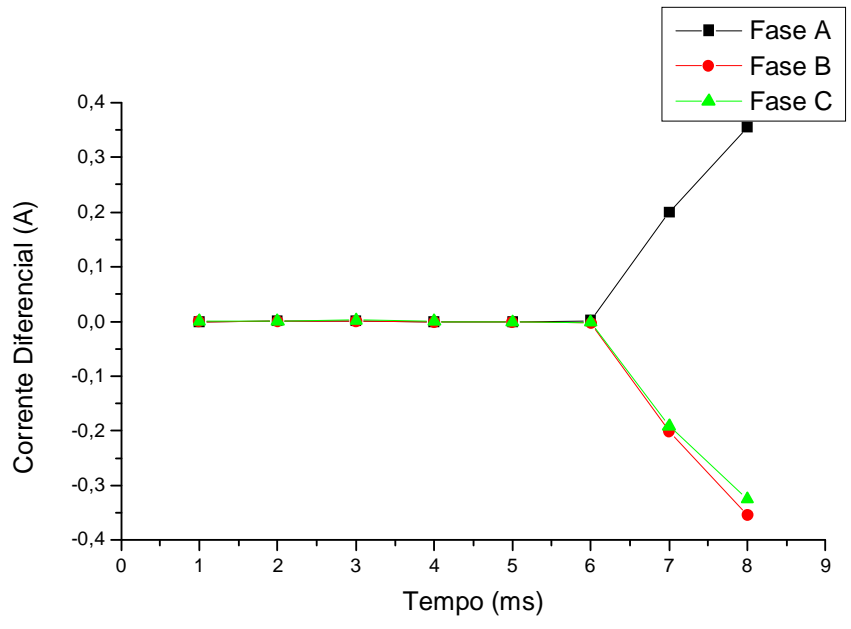

Figura 15: Sinal de falta interna distorcido pela saturação

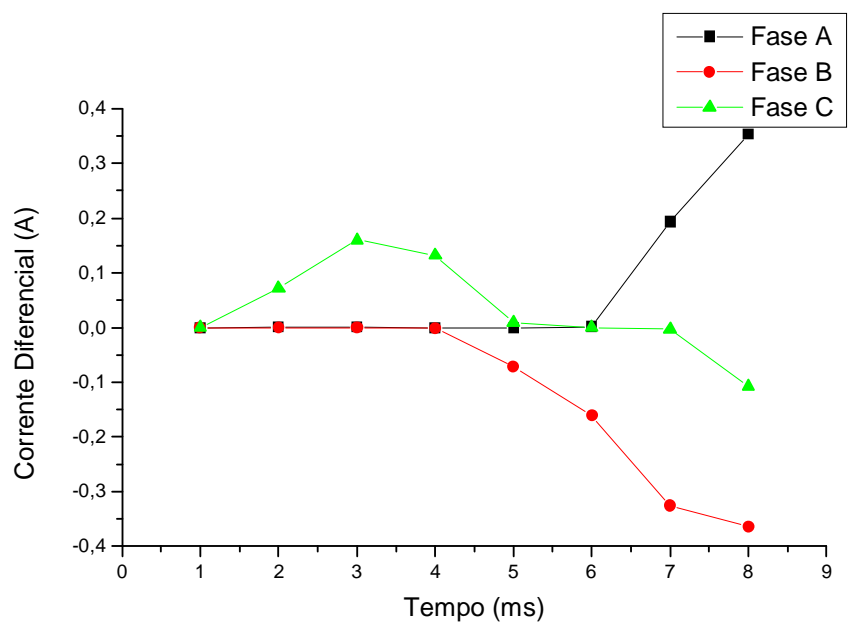

Figura 16: Sinal de falta interna com TC ideal

\section{CONCLUSÕES}

Este trabalho apresentou uma técnica alternativa para a correção de ondas distorcidas provenientes da saturação dos dispositivos transformadores de corrente através das ferramentas inteligentes baseadas em Redes Neurais Artificiais Recorrentes. Os TCs estão presentes em sistemas elétricos de potência com a finalidade de proteção e medição, sendo altamente suscetíveis à saturação. O programa EMTP-ATP foi escolhido como ferramenta computacional para a simulação de um transformador de potência utilizado na geração de dados de treinamento e testes para as RNAs. Muitas arquiteturas de redes neurais artificiais foram treinadas e testadas, sendo que as melhores configurações obtidas e mostradas foram as arquiteturas recorrentes de Elman/Jordan.

Após o processo de treinamento e testes das redes neurais, 


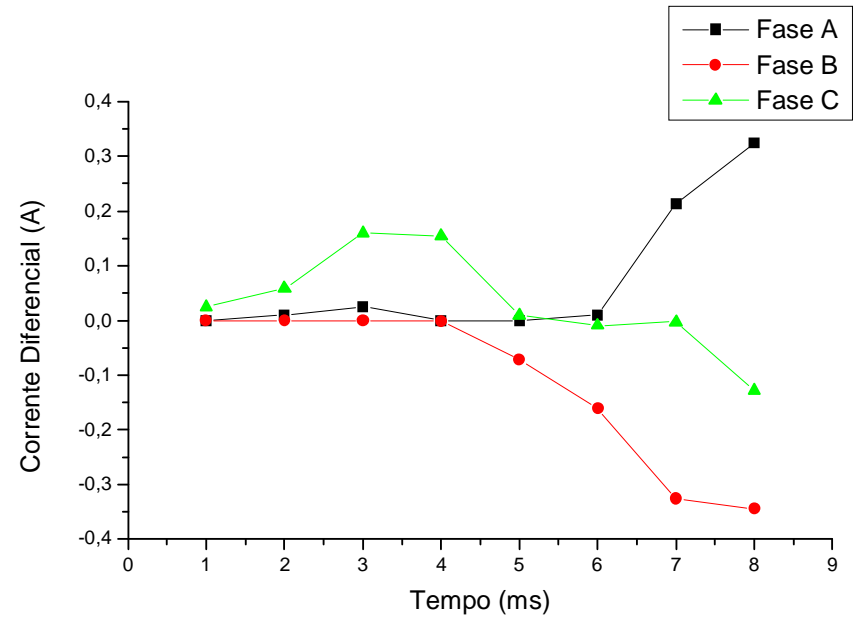

Figura 17: Sinal de falta interna reconstruído pela RNA

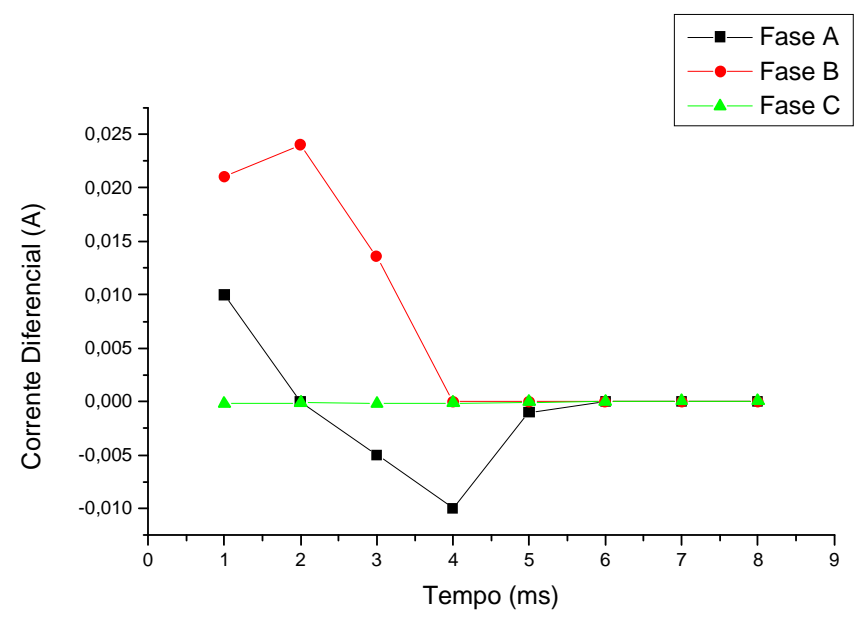

Figura 18: Sinal de falta interna distorcido pela saturação

a melhor estrutura obtida e apresentada neste trabalho a fim de reconstruir as formas de onda dos sinais diferenciais distorcidos pela saturação dos TCs foi a estrutura tipo Elman de topologia $24+20+24$, ou seja, 24 neurônios na camada de entrada, 20 neurônios na camada oculta, 24 neurônios na camada recorrente e 24 células na camada de saída, a qual reconstruiu o sinal. Esta configuração obteve convergência mais rápida e resultados de testes precisos.

O trabalho proposto não necessitou de pré-processamentos dos sinais, sendo que a utilização de RNAs recorrentes asseguraram um processo de convergência mais rápido e mais preciso, se comparado a outras estruturas neurais já estudadas.

A utilização de configurações de RNAs do tipo Elman possibilitou a reconstrução direta dos sinais de correntes diferenciais que serão utilizadas pelo dispositivo de proteção.

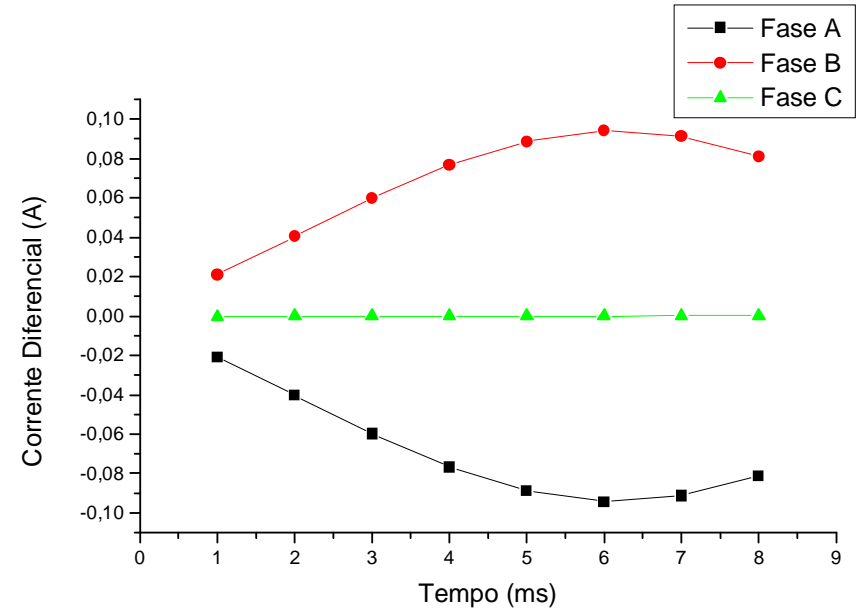

Figura 19: Sinal de falta interna com TC ideal

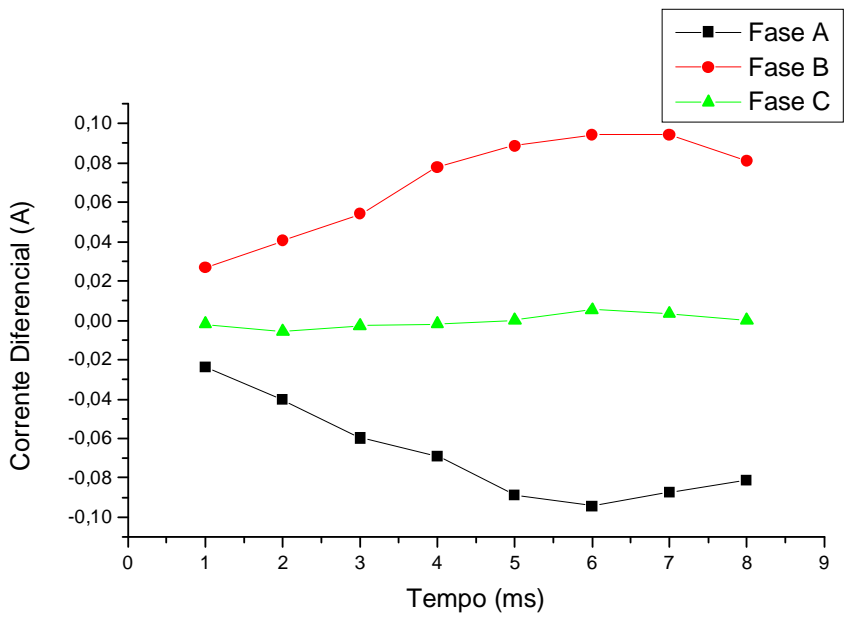

Figura 20: Sinal de falta interna reconstruído pela RNA

De forma geral, o método desenvolvido mostrou-se eficiente em busca do objetivo proposto. Resultados promissores relativos à aplicação são apresentados.

A aplicação da ferramenta Redes Neurais Artificiais constitui, portanto, uma nova e importante etapa na metodologia de análise de sinais oriundos de transformadores de corrente na busca de um melhor desempenho de funções como proteção, medição e oscilografia de Sistemas Elétricos de Potência.

\section{REFERÊNCIAS}

Alternative Transients Program Rule Book (1987), Leuven EMTP Center.

Barnett, C.W. ; Chadwick, J.W. et al. (1993). Relay Performance Considerations with Low Ratio CTs and High 
Fault Currents, IEEE Transactions on Power Delivery, v.8, n.3, pp. 884-887.

Bozoki, B. and C.M. Calhoun (1990). Gapped Core Transformer Characteristics and Performance, IEEE Power System Relaying Committee, IEEE Transactions PWRD, v.5, n.4, pp. 1732-1740.

Bradley, D.A. ; Gray, C.B. and D. O'Kelly (1978). Transients Compensation of Current Transformers, IEEE Transactions PAS, v.97, n.4, pp. 1264-1271.

Carvalho, A.C.P.L.F. ; Braga, A.P. and T.B. Ludermir (1998). Fundamentos de Redes Neurais Artificiais, XI Escola Brasileira de Computação.

Chen, K.W. and S.T. Glad (1991). Estimation of the Primary Current in a Saturated Transformer, Proceedings of the IEEE Conference on Decision and Control, pp. 116124.

Conrad, T. ; Schlabbach, J. and R. Speh (1984). Verfahren zur Korrektur der verzerrten Sekundarstrome von Stromwandlern, Electrotechnische Zeitschrift Archiv, v.6, n.2, pp. 77-79.

Coury, D.V.; Campos, P.G. and M.C. Tavares (1998). Modelling a Power Transformer for Investigation of Digital Protection Schemes. 8th International Conference on Harmonics and Quality of Power (ICHQP'98), pp. 489-494.

Cummins, J.C. ; Yu, D.C. and L.A. Kojovic (2000). Simplified Artificial Neural Network Structure with the Current Transformer Saturation Detector provides a Good Estimate of Primary Currents, 2000 Power Engineering Society Summer Meeting, v.3.

Elman, J.L. (1990). Finding Structure in Time. Cognitive Science, $\mathrm{n} .14$, pp.179-211.

Glinkowski, M.T. and J. Esztergalyos (1996). Transient Modeling of Electromechanical Relays, Part 1: Armature Type Overcurrent Relay, IEEE Transactions on Power Delivery, v.11, n.2.

Horowitz, S. H. and A.G. Phadke (1995). Power System Relaying, Research Studies Press Ltd., 2nd ed., England.

Hosemann, G. and H.M. Steigerwald (1993). Modal Saturation Detector for Digital Differential Protection, IEEE Transactions on Power Delivery, v.8, n.3, pp. 933-940.

IEEE Power System Relaying Committee (1997). Transient Response of Current Transformers, IEEE Transactions $P A S$, v.96, n.6, pp.1809-1814.

IEEE (1991). IEEE Guides and Standards for Protective Relaying Systems, Spring.
Jamali, S. and K.M. Radji (1997). A Digital Compensating Technique for the Saturation of Protective Current Transformers, Proceedings of Thirty Second Universities Power Engineering Conference, v.1, pp. 10-12.

Jordan, M.I. (1986). Attractor Dynamics and Parallelism in a Connectionist Sequential Machine. Proc. of the Eighth Annual Conf. of the Cognitive Science Society, pp. 531546.

Kang, Y.C. ; Kang, S.H. ; Park, J.K. ; Johns, A.T. and R.K. Aggarwal (1996). Development in Hardware Implementation of a Compensating Algorithm for the Secondary Current of Current Transformers, IEEE Proceedings in Electric Power Applications, v.143, n.1, pp. 41-49.

Kang, Y.C. ; Kang, S.H. ; Park, J.K. ; Johns, A.T. and R.K. Aggarwal (1997). Algorithm for Compensating Secondary Currents of Current Transformers, IEEE Transactions on Power Delivery, v.12, n.1, p. 116-124.

Kezunovic, M. ; Kojovic, L.A. ; Fromen, C.W. ; Sevcik, D.R. and F. Phillips (1993). Experimental Evaluation of EMTP-based Current Transformer Models for Protective Relay Transient Study, 93 WM 041-4 PWRD, IEEE/PES Winter Meeting, New York.

Masson, L. (1979). Circuit for the Dynamic Control of Magnetic Flux in Current Transformers, IEEE Transactions PAS, v.98, n.6, pp. 1990-1995.

McCulloch, W. S. and W. Pitts (1943). A Logical Calculus of the Ideas Immanent in Nervous Activity. Bulletin of Mathematical Biophysics, n.5, pp. 115-133.

Mikrut, M. ; Winkler, W. and B. Witek (1989). Performance of Differential Protection for Three-winding Power Transformers During Transient CTs Saturation, 4th International Conference on Developments in Power System Protection, IEE Pub. n.302, pp. 40-44.

Minsky, M. and S. Papert (1969). Perceptrons: an Introduction to Computational Geometry. MIT Press, Massachussets.

Pihler, J., Grcar, B., Dolinar, D. (1997). Improved Operation of Power Transformer Protection using Artificial Neural Network, IEEE Transactions on Power Delivery, v.12, n.3., pp.1128-1136.

Powell, L.J. (1979). Current Transformer Burden and Saturation, IEEE Transactions IA, v.15, n.3, pp. 294-302.

Requirements for Instrument Transformers (1993), C57.13 ANSI/IEEE Standard. 
Rosenblatt, F. (1958). The Perceptron: A Probabilistic Model for Information Storage and Organization in the Brain. Psychol. Rev. , n.65, pp. 386-408.

Rumelhart, D. E., Hinton, G. E. and R. J. Williams (1986). Learning Representations by Back-propagation Errors, Nature, n.323, pp. 533-536.

Segatto, E.C., Coury, D.V. (2005). A Power Transformer Protection with Recurrent ANN Saturation Correction, IEEE Power Engineering Society General Meeting, San Francisco, USA.

Segatto, E.C., Coury, D.V. (2006). A Differential Relay for Power Transformers Using Intelligent Tools, IEEE Transactions on Power Systems, v.21, n.3, pp.11541162.

Sidhu, T.S. ; Gill, H.S. and M.S. Sachdev (1999). A Power Transformer Protection Technique with Stability during Current Transformer Saturation and Ratio-mismatch Conditions, IEEE Transactions on Power Delivery, v.14, n.3, pp. 798-804.

SNNS User Manual (1995). Institute for Parallel and Distributed High Performance Systems, University of Stuttgard.

Wright, A. (1968). Current Transformers, their Transient and Steady State Performance, London: Chapman \& Hall, p. 77.

Yu, D.C. ; Wang, Z. ; Cummins, J.C. ; Yoon, H.J. and L.A. Kojovic (1999). Neural Network for Current Transformer Saturation Correction, Proceedings of IEEE Power Engineering Society - Transmission and Distribution Conference, v.1, pp. 441-446.

Yu, D.C. ; Cummins, J.C. ; Wang, Z. ; Yoon, H.J. and L.A. Kojovic (2001). Correction of Current Transformer Distorted Secondary Currents due to Saturation using Artificial Neural Networks, IEEE Transactions on Power Delivery, v.16, n.2, pp.189-194. 